\title{
BIENESTAR EMOCIONAL E INTELIGENCIA EN LA PUBERTAD Y LA ADOLESCENCIA
}

\author{
Vicente Pelechano \\ Wenceslao Peñate \\ Gustavo Ramírez \\ Francisco Díaz Cruz
}

\section{RESUMEN}

Sobre una muestra de 117 participantes (púberes $y$ adolescentes) se evaluaron los componentes propuestos de bienestar emocional (afectividad -PANAS-C-y satisfacción con la vida -SLSS-) e inteligencia (Cattell). La satisfacción con la vida se asocia con los factores de afectividad pero no con inteligencia. La llamada afectividad positiva y la negativa mantienen entre sí relaciones dispares a lo largo de este período evolutivo. Los resultados suponen una limitación a la teoría evolutiva de la diferenciación progresiva y el modelo de bienestar emocional en general y sugieren algunas reflexiones acerca del desarrollo evolutivo en estos dominios del funcionamiento personal.

Paiabras clave: BIENESTAR EMOCIONAL, AFECTIVIDAD POSITIVA Y NEGATIVA, SATISFACCIÓN CON LA VIDA EN ADOLESCENCIA, EVOLUCIÓN DE INTELIGENCIA Y AFECTIVIDAD.

Correspondencia: Vicente Pelechano. Departamento de Personalidad Evaluación y Tratamientos Psicológicos de la Universidad de La Laguna (Tenerífe), correo electrónico: vpelecha ull.es 


\section{SUMMARY}

The components of the emotional well-being (life satisfaction, positive affect and negative affect) have been tested in a sample of Spanish pre-adolescent and adolescents. Also Cattell general inteligence test has been fulfilled. Positive and negative affect are related to life satisfaction but not with intelligence. Positive affect and negative affect show correlations non-coherent among them. The results are discussed in terms of the general developmental discrimination hypothesis and the bipolarity on positive and negative affects. The nature of the variables under study and their consolidation level are in order to offer an interpretation according to parameter's model.

Key words: EMOTIONAL WELL-BEING, POSITIVE AFFECT AND NEGATIVE AFFECT IN ADOLESCENCE, DEVELOPMENT OF INTELLIGENCE AND AFFECT.

Aunque pueda parecer obvio, este trabajo comienza reivindicando no solamente la conveniencia, sino también la necesidad de que la psicología estudie las vivencias y los sentimientos humanos, sus vivencias y los determinantes y posibilitantes tanto del malestar como del bienestar personal, frente al reduccionismo cientista que pretende reducir la psicología poco menos que al estudio de los registros fisiológicos y los tiempos de reacción ante la presentación de estímulos simples. $Y$ esta reivindicación resulta importante en la medida en que se comienzan a vislumbrar movimientos "oficiales" en los que se defiende que la psicología debe reducirse a una radical fisiología de aficionados sin análisis de los contenidos psicológicos. Pensamos que el reduccionismo científico en psicologia, propio de fases históricas pasadas, está rebrotando en aras de un cientismo descerebrado que ni llevó ni puede llevar a la psicología a utilidad social y personal alguna.

Uno de los objetivos que persiguió M. E. P. Seligman en su presidencia de la American Psychological Association en los últimos años del siglo $X X$, fue la promoción del estudio de los aspectos "positivos" del funcionamiento humano. Inteligencia, creatividad, 
sabiduría, emocionalidad positiva, optimismo, flexibilidad, altruismo, felicidad y bienestar se han ido consolidando como dominios que han dado ya sus frutos incluso en un nivel de manual introductorio y de evaluación (Argyle, 2001; Aspingall \& Staudinger, 2003; López \& Zinder; 2003), lo que ha dado lugar a una especialización discutida aunque creciente de "psicologla positiva". Dentro de esta sensibilidad han preocupado con especial insistencia las percepciones que las personas tienen de su existencia 0 , dicho de otra manera, sü punto de vista respecto a su experiencia de la vida, que se ha plasmado en dos líneas de trabajo: la primera se ocupa del grado de los sentimientos positivos experimentados (relacionados estrechamente con la felicidad) y de las percepciones que se acompañan a estos sentimientos (la satisfacción o componentes cognitivos de la felicidad), lo que se ha relacionado con el bienestar emocional; la segunda línea se ocupa de las dimensiones de funcionamiento positivo (Jahoda, 1958; Keyes, 1998; Ryff \& Keyes, 1995). El estudio del bienestar emocional, como una parte del bienestar subjetivo ocupa un lugar prominente en esta orientación y una de las líneas de trabajo defiende que el bienestar subjetivo se encuentra formado por tres elementos básicos: la satisfacción con la vida, el afecto positivo y el afecto negativo (Bryant \& Veroff, 1982; Keyes \& Magyar-Moe, 2003; Lucas, Diener \& Suh, 1996; Shmotkin, 1998), o de manera más precisa; el equilibrio entre el afecto positivo y el afecto negativo.

El caso es que esta propuesta ha sido valìdada básicamente en poblaciones adultas $y$ en las que no se ha tomado en cuenta la inteligencia ni abundan los datos evolutivos acerca de si o no estos elementos constitutivos del bienestar emocional aparecen antes de la fase adulta.

\section{Afectividad}

Pese a la existencia de tradiciones consolidadas en el estudio de la emoción, la verdad es que no parece existir una definición consensuada al respecto. Frida (1999) distingue distintos componentes: afecto o experiencia de placer o dolor, estimación de un objeto o suceso como bueno o malo, preparación para una acclón determinada sobre el ambiente, activación neurovegetativa y cambios cognitivos. Ortony y 
Turner (1990) incluyeron estados afectivos sin valencia clara, como sorpresa, interés y deseo (pueden ser positivos, negativos o afectivamente neutrales). $Y$ el intento de Frida ha sido calificado como una descripción de posibles componentes, aunque no una definición (Lucas, Diener \& Larsen, 2003). Asimismo existe una variabilidad en la utilización del lenguaje que se refiere al tema. Siguiendo la mayoria de autores y coherentemente con el modelo de parámetros se diferenciará entre emoción (temporalmente puntual y referido a uno $u$ otro estado psicológico), estado de ánimo (contextual de duración y generalización intermedia) y sentimiento-dimensión temperamental (referido a aspecto psicológico duradero y de amplia generalización que guardaria relaciones estrechas con las dimensiones temperamentales de la personalidad: el denominado "afecto negativo" estaría relacionado, entre otros, con neuroticismo, depresión, hostilidad y autoritarismo mientras que el "afecto positivo" tendría relación con dimensiones tales como extraversión social y cordialidad.

Esta opción supone la existencia de relaciones y de cierta estructura jerarquizada de las emociones y afectos humanos, de la que existe un modelo dimensional directo y otro circumplejo. En este último se defiende una estructura de círculo alrededor de un punto formado por la intersección de dos dimensiones independientes (Larsen \& Diener, 1992; Watson, Wiese, Vaidya \& Tellegen, 1999). Pese a la propuesta de una estructura tridimensional (Schimmack \& Grob, 2000), existe un mayor acuerdo entre los investigadores respecto a una estructura bidimensional, aunque la denominación concreta de las dimensiones propuestas varía: el grupo de Russell (Russell, 1980, Russell \& Feldman Barreto, 1999) propone placer y activación; Watson y Tellegen (1985, Watson et al., 1999) proponen rotar los ejes $45^{\mathcal{Q}}$ para obtener dos factores independientes: afecto positivo (combinación de afecto placentero alto y activación alta) 0 activación positiva y afecto negativo o activación negativa (afecto displacentero alto y activación alta). Por lo que se refiere a las emociones positivas, aunque el número de las mismas puede variar de dos a cuatro, se acepta que estas emociones presentan relaciones muy altas entre sí.

Existen, al menos, tres intentos explicativos respecto a la dependencia $o$ independencia entre las emociones positivas y las negativas: la teoría contextual de los afectos (Zautra, Potter y Reich, 
1997), la teoría del yo respecto del cambio subjetivo y la salud mental (Keyes y Riff, 2000) y la opción de parámetros de Pelechano (1996, $1989,2000)$, relacionada, en parte, con la sugerencia de Diener sobre referencias temporales en la cumplimentación de los instrumentos. En la contextuación del afecto se defiende que la relación es mayor cuanto mayor volumen de estrés (hipótesis relacionada, a su vez, con una menor diferenciación a mayor volumen de activación); en la teoría del sí mismo se asume que los humanos perciben el cambio personal enfrentándose a los estándares personales que poseen y de esta manera deciden si la información es buena, mala o una mezcla de las dos (la sensibilidad a cambio afectivo es distinta según se perciba un mantenimiento, una disminución o un incremento); en la teoría de parámetros la relación existente entre dos o más variables depende tanto del contenido psicológico de las mismas, como del nivel de consolidación de las variables (los estados pueden ser modificados con mayor facilidad que los rasgos básicos tanto en sus puntuaciones como en sus relaciones; las relaciones entre las variables no necesitan ser invariantes dependiendo, en parte, de las condiciones en la recogida de información; aunque, en todo caso, se propone una lógica no dicotómica en contenidos psicológicos $y$, en especial, por lo que se refiere a afectos (se puede odiar y amar a la vez).

Estas consideraciones sugieren la conveniencia de recoger información cerca de afectos positivos y negativos en el estudio del bienestar emocional. Por otro lado, la recogida de información en situaciones de vida diaria arrojaría alguna luz respecto a algunas de las predicciones de las teorías acerca de la relación entre afectos $y$, finalmente, la participación de personas en fases evolutivas anteriores a la fase adulta permitirá pergeñar hipótesis y sugerencias respecto a la evolución de la afectividad. Desde hace muchos años se ha sugerido y prácticamente asumido, que la evolución psicológica de los afectos va de una primitiva indiferenciación (recién nacido) a una progresiva diferenciación de afectos y/o emociones. Esta hipótesis genérica se sigue manteniendo a sabiendas de su generalidad e inespecificidad: junto a las diferenciaciones se dan integraciones y reestructuraciones que "simplifican" las estructuras que se van obteniendo. $Y$ es posible, además, que exista más de un camino en la evolución de los afectos y de las estructuras afectivas, por lo que la diferenciación acerca de 
afectos positivos y negativos en forma de exclusión sea un primer paso en la organización afectiva, pero puede adolecer de insuficiencias graves.

\section{Satisfacclón con la vida}

El segundo elemento importante aducido en el bienestar emocional es un elemento cognitivo: la satisfacción con la vida. Se trata de una estimación respecto al nivel de satisfacción personal que un ser humano tiene sobre la vida que ha vivido hasta ese momento.

Las primeras formas de evaluación consistieron en una única cuestión en la que se pedia el grado de satisfacción general con la vida hasta ese momento, con distintas posibilidades de respuesta: puntuaciones de 0 a $7,9,100$, etc. Asimismo se han utilizado escalas gráficas "globales". Posteriormente se han propuesto instrumentos en los que se exponen distintas preguntas respecto a la satisfacción en distintos contextos de vida y evolución de esta satisfacción (rememoración).

En una primera consideración debería ir acompañado de afectividad "positiva", aunque es posible pensar que pueden encontrarse presente elementos de afectividad "negativa", como indicadores de aspectos perturbadores del funcionamiento personal aunque con la sensación de poder ser superados; incluso pueden darse relaciones positivas entre afectividad negativa y sentido de la vida en la medida en que pese a la existencia de afectos negativos (entendidos como indicadores de "realidad objetiva" problemática), se puede tener una percepción de una vida con pleno sentido (incluso con valoración positiva). Además, en casos en los que exista una sintonía entre el yo y la conducta realizada (incluso los estilos de conducta) como sucede con los trastornos de personalidad, es posible alcanzar puntuaciones altas en la satisfacción con la vida y con afectividad cargada de valencia "negativa" desde fuentes externas (piénsese, por ejemplo, en el trastorno antisocial o disocial de la personalidad).

No existen muchos datos respecto a consideraciones evolutivas de la relación entre afectividad y satisfacción con la vida, aunque la tendencia general observable sería la de una mayor relación entre las 
dos valencias de afectividad y satisfacción con la vida en la pubertadadolescencia en función de la hipótesis general de la diferenciación.

\section{El papel de la inteligencia}

En buena cuenta el papel de la inteligencia general e incluso tipo cociente intelectual en el funcionamiento y estructura de los afectos se supone irrelevante, aunque la cuestión dista mucho de estar resuelta. Por una parte, en el trabajo de revisión de Isen (2003 se defiende el papel favorecedor de los afectos positivos en el rendimiento ante distintas tareas cognitivas, pero ninguna de ellas recoge el tipo de tareas y/o problemas que se encuentran en los tests de inteligencias tradicionales. Por otro lado, Sternberg (2003), entre otros, viene defendiendo la necesidad de redefinir el concepto y los instrumentos de medida de la inteligencia, en términos de "inteligencia con éxito" y que recogería aspectos académicos no académicos de la misma, con elementos de papel y lápiz y otros de corte más social e instrumental. Es en estas ampliaciones de tareas y recursos de medida en donde se ubican las relaciones de la inteligencia con la afectividad y no en condiciones "normales" pruebas de papel y lápiz.

Posiblemente la selección de conceptos y tareas recoge la división tripartita del psiquismo humano, en cognición, volición y afecto y los renovados esfuerzos por estudiar cada una de estas partes de forma independiente de las otras con el fin de poder profundizar en su estudio. Evolutivamente sin embargo, tampoco existen datos que permitan formular hipótesis concretas, mucho más allá de la diferenciación y, en este caso, cabría esperar relaciones escasas aunque mayores en los más pequeños que en los mayores.

En todo caso, en la medida en que se trate de condiciones de medida de vida cotidiana, sin estresores intensos y con una duración de evaluación corta, no cabe esperar relaciones intensas.

\section{MÉTODO}

(a). Participantes: 117 escolares correspondientes al un centro escolar público del municipio de Arona, en el sur de la isla de Tenerife (Islas Canarias) fueron los participantes en este estudio. 42 
correspondieron a $6^{0}$ de Educación Primaria, 41 a $1^{9}$ de la Educación Secundaria Obligatoria (ESO), y 34 a $2^{\circ}$ de la ESO. Por olo que se refiere al género, un $51,3 \%$ eran niños (60) y un $48,7 \%$ (57) eran niñas. El rango de edad cronológica fue de 11 a 14 años, distribuidos de la manera siguiente: 27 alumnos de 11 años, 31 de 12 años, 30 de 13 años y 29 de 14 años.

(b). Instrumentos: Para evaluar el nivel de inteligencia se ha utilizado la escala de factor $g$ de R. B. Cattell (1931). Para evaluar la afectividad positiva y negativa se ha utilizado la versión infanto-juvenil de la Positive and Negative Affect Scale (Laurent, Catanzaro, Joiner Jr., Rudoph, Potter, Lambert, Osborne y Gathright, 1999), y para evaluar la satisfacción vital se ha utilizado la Satisfaction Life Student Scale (Huebner, 1991).

Escala Factor $g$ de Cattell, escala 2A. Esta escala es una de las desarrolladas por Cattell (1931), con la finalidad de proporcionar una evaluación rápida del factor g como medida del Cociente Intelectual (Cl), que estuviera libre de elementos verbales y culturales. Se correspondería con la idea de inteligencia fluida de este autor, con una influencia clara de aspectos biológicos (y genéticos) en su conformación. Consta de cuatro subescalas: series, clasificación, matrices y condiciones, y tiene un tiempo de aplicación fijado (12 minutos y medio). Con respecto a la bondad psicométrica, la adaptación española (TEA, 1973) muestra unos índices de consistencia interna elevados y una buena validez convergente con otras pruebas de inteligencia. En nuestro caso, hemos utilizado esta escala a modo de screening para detectar un nivel de funcionamiento intelectual básico.

Positive and Negative Affect Scale-C (PANAS-C). Esta escala evalúa la afectividad positiva y negativa para niños y adolescentes. Ha sido elaborada por Laurent y cols., (1999), basada en el modelo teórico y la escala original para adultos (PANAS, Watson, Clark y Tellegen, 1988). Fundamentada en la teoría general del afecto (activacióndesactivación y placer-displacer), su elaboración ha tenido como fundamento la diferenciación entre ansiedad y depresión en función de esos dos tipos de afectos. Esta escala ha sido adaptada al español por Sandín (2003). La escala consta de 30 ítems/adjetivos afectivos que se deben responder de acuerdo a cinco alternativas de acuerdo a cómo se ha sentido en las últimas semanas (desde 'en absoluto' hasta 
'extremadamente'). La escala permite una medida general de afectividad positiva y otra de afectividad negativa. Secundariamente permite obtener medidas más básicas, pudiéndose obtener índices por separado de los dos componentes de la afectividad positiva: placer/ activación (alegre) placer/desactivación (tranquilo); y de los dos componentes de la afectividad negativa: displacer/activación (nervioso) y displacer/desactivación (triste). Los datos tanto de la versión original como la adaptación española presentan unos índices aceptables de ajuste con respecto al modelo PANAS, una buena consistencia interna para las dos medidas generales de afecto, una buena validez convergente y una buena validez diferencial para discriminar entre estados de ansiedad y de depresión.

Satisfaction Life Student Scale (SLSS). Esta escala ha sido elaborada por Huebner (1991) con la finalidad de obtener un índice general de satisfacción vital o bienestar subjetivo basado en el modelo propuesto por Diener (1984). Esta escala consta de 30 items. Algunos de ellos tienen un alto nivel de contagio con las escalas PANAS ('me siento triste', 'tengo miedos', 'estoy animado'...), por lo que en este estudio sólo se utilizaron siete de los ítems que más genéricamente están relacionados con la satisfacción general, tal y como propone el propio autor (Huebner, 1991, 1994): 'mi vida va bien', 'mi vida es ideal', 'me gustarla cambiar algunas cosas en mi vida' (negativo), 'me gustaría tener un tipo de vida diferente' (negativo), 'tengo una vida buena', 'tengo lo que quiero en la vida', y 'mi vida es mejor que la de la mayoría de los niños'. Los datos psicométricos, tanto de la versión original como de la adaptación española (Alsinet, 1998; Rosich, 1999; referenciada en Pestana, Rosich y Codina, 2003), apoyan la existencia de un índice general de bienestar con una elevada consistencia interna.

(c). Procedimiento: Como se ha indicado más atrás, se quiso contar con una muestra de chicos y chicas que evolutivamente representaran esas primeras fases puberales y adolescentes de desarrollo afectivo y cognitivo. Para ello, se contactó con el centro escolar para evaluar al alumnado de los tres últimos cursos ( $6^{9}$ de educación primaria, $1^{q}$ y $2^{Q}$ de la ESO). Una vez obtenida la autorización, se mantuvo una reunión con los tutores de cada curso (dos por nivel), se les explicó el sentido de la investigación y la finalidad de la misma. Posteriormente se les instruyó para que administraran las pruebas. La prueba de inteligencia 
fue administrada por una misma tutora, a la que se formó en la administración de las pruebas, que fueron cumplimentadas en días distintos, aunque siempre en el mismo orden. La primera prueba fue la de inteligencia, la segunda, la de PANAS-C y la última la SLSS.

De la muestra final fueron eliminados los alumnos con adaptaciones curriculares significativas y aquellos otros que, a juicio de los tutores, no mantuvieron un comportamiento atento a la tarea a realizar. Finalmente se eliminaron aquellos que tenían más de 14 años. Así, la muestra final quedó constituida por 117 escolares.

\section{RESULTADOS}

Los primeros análisis realizados se llevaron a cabo para conocer si las variables de género y edad estaban modulando las puntuaciones obtenidas en las medidas cognitivas, afectivas y de satisfacción con la vida. Para ello se llevó a cabo un análisis de varianza tomando como variables independientes al género y la edad y como variables dependientes a las puntuaciones en satisfacción vital, inteligencia, afecto positivo, afecto negativo y las puntuaciones desagregadas de los dos tipos de afecto en función de la valencia y la activación: placer/ activación, placer/desactivación, displacer/activación y displacer/ desactivación'. En la tabla 1 se resumen los resultados del análisis para el género y la edad.

Como puede observarse, a pesar de que los chicos tienden a puntuar más que las chicas en casi todas las variables (salvo en satisfacción con la vida), ninguno de los contrastes ha alcanzado el nivel de significación estadística, poniendo de manifiesto que las variables evaluadas no estaban afectadas por el género. Sin embargo, en el caso de la edad se observan dos contrastes significativos: para la afectividad positiva y para la variable placer/activación. La afectividad positiva tiende a disminuir con la edad de manera apreciable. De los dos componentes desagregados de la afectividad positiva, es la variable activa (placer/activación) la que posee mayor peso, mostrando claramente su disminución a medida que se aumenta la edad.

\footnotetext{
1 En este caso, dado que estas cuatro variables no contaban con el mismo número de items, las puntuaciones totales se transformaron en una escala de 1 a 5 .
} 
Un segundo grupo de análisis se llevó a cabo para conocer las relaciones que mantenían entre sí las puntuaciones en afecto, inteligencia y en el índice de satisfacción con la vida. Para ello se llevó a cabo un análisis correlacional (tipo Pearson) entre las distintas variables. En la tabla 2 se encuentran los coeficientes obtenidos.

Las correlaciones de la inteligencia con la afectividad positiva y la afectividad negativa mostraron una clara independencia entre ambas variables (coeficientes de 0,09 y $-0,09$ respectivamente), en contra de lo esperado desde aquellas posiciones que apoyarían una evolución afectiva y cognitiva común. Estos coeficientes no se modifican apreciablemente cuando se toman en cuenta los elementos más específicos del afecto: ni el placer ni la actividad mejoran significativamente los coeficientes anteriores. Por otro lado, las

Tabla 1. Resumen de los análisis de varianza para género y los cuatro grupos de edades sobre las variables de satisfacción vital, afecto y $C . I$

\section{GÉNERO}

\begin{tabular}{|c|c|c|c|c|c|c|c|}
\hline & Género & $\mathbf{N}$ & Media & D.T. & gl & $\mathrm{F}$ & Sig. \\
\hline \multirow{2}{*}{$\mathrm{Cl}$ (inteligencia) } & Chicos & 60 & 99,27 & 17,90 & \multirow{2}{*}{1,115} & \multirow{2}{*}{2,60} & \multirow{2}{*}{0,110} \\
\hline & Chicas & 57 & 93,88 & 18,28 & & & \\
\hline \multirow{2}{*}{ AFECTO POSITIVO } & Chicos & 60 & 55,57 & 9,19 & \multirow{2}{*}{1,115} & \multirow[t]{2}{*}{1,65} & \multirow{2}{*}{0,202} \\
\hline & Chicas & 57 & 53,29 & 7,39 & & & \\
\hline \multirow[t]{2}{*}{ AFECTO NEGATIVO } & Chicos & 60 & 26,75 & 8,24 & \multirow{2}{*}{1,115} & \multirow{2}{*}{1,60} & \multirow{2}{*}{0,209} \\
\hline & Chicas & 57 & 24,89 & 5,95 & & & \\
\hline \multirow{2}{*}{ PLACERACTIVACION } & Chicos & 60 & 3,79 & 0,60 & \multirow{2}{*}{1,115} & \multirow{2}{*}{1,37} & \multirow{2}{*}{0,245} \\
\hline & Chicas & 57 & 3,65 & 0,50 & & & \\
\hline \multirow[t]{2}{*}{ DISPLACER/ACTIVACIÓN } & Chicos & 60 & 1,92 & 0,58 & \multirow{2}{*}{1,115} & \multirow{2}{*}{2,29} & \multirow{2}{*}{0,134} \\
\hline & Chicas & 57 & 1,75 & 0,48 & & & \\
\hline \multirow[t]{2}{*}{ PLACERIDESACTIVACIÓN } & Chicos & 60 & 2,65 & 0.91 & \multirow{2}{*}{1,115} & \multirow{2}{*}{2,88} & \multirow{2}{*}{0,092} \\
\hline & Chicas & 57 & 2,37 & 0,85 & & & \\
\hline \multirow[t]{2}{*}{ DISPLACER/DESACTIVACIÓN } & Chicos & 60 & 1,72 & 0,66 & \multirow{2}{*}{1,115} & \multirow{2}{*}{0,81} & \multirow{2}{*}{0,370} \\
\hline & Chicas & 57 & 1,62 & 0,51 & & & \\
\hline \multirow{2}{*}{ SATISFACCIÓN } & Chicos & 60 & 20,53 & 4,16 & \multirow{2}{*}{1,115} & \multirow{2}{*}{1,185} & \multirow{2}{*}{0,279} \\
\hline & Chicas & 57 & 21,35 & 3,76 & & & \\
\hline
\end{tabular}




\section{EDAD}

\begin{tabular}{|c|c|c|c|c|c|c|c|}
\hline & edad & $\mathrm{N}$ & Madia & D.T. & gl & $\mathrm{F}$ & Sig. \\
\hline \multirow{4}{*}{$\mathrm{Cl}$ (inteligencia) } & 11 & 27 & 98,59 & 17,69 & \multirow{4}{*}{3,113} & \multirow{4}{*}{2,05} & \multirow{4}{*}{0,100} \\
\hline & 12 & 31 & 95,48 & 16,68 & & & \\
\hline & 13 & 30 & 93,83 & 16,01 & & & \\
\hline & 14 & 29 & 99,66 & 17,96 & & & \\
\hline \multirow{4}{*}{ AFECTO POSITIVO } & 11 & 27 & 57,43 & 7,79 & \multirow{4}{*}{3,113} & \multirow{4}{*}{3,12} & \multirow{4}{*}{0,030} \\
\hline & 12 & 31 & 56.72 & 8,63 & & & \\
\hline & 13 & 30 & 53,00 & 8,35 & & & \\
\hline & 14 & 29 & 50,85 & 7,79 & & & \\
\hline \multirow{4}{*}{ AFECTO NEGATIVO } & 11 & 27 & 26,53 & 6,02 & \multirow{4}{*}{3,113} & \multirow{4}{*}{2,16} & \multirow{4}{*}{0,098} \\
\hline & 12 & 31 & 28,41 & 8,53 & & & \\
\hline & 13 & 30 & 25,07 & 7,21 & & & \\
\hline & 14 & 29 & 23,67 & 6,06 & & & \\
\hline \multirow{4}{*}{ PLACER/ACTIVACION } & 11 & 27 & 3,96 & 0,54 & \multirow{4}{*}{3,113} & \multirow{4}{*}{3,66} & \multirow{4}{*}{0,015} \\
\hline & 12 & 31 & 3,85 & 0,57 & & & \\
\hline & 13 & 30 & 3,62 & 0,52 & & & \\
\hline & 14 & 29 & 3,48 & 0.49 & & & \\
\hline \multirow{4}{*}{ DISPLACER/ACTIVACIÓN } & 11 & 27 & 1,75 & 0.52 & \multirow{4}{*}{3,113} & \multirow{4}{*}{2,00} & \multirow{4}{*}{0.119} \\
\hline & 12 & 31 & 2,05 & 0,61 & & & \\
\hline & 13 & 30 & 1,77 & 0,54 & & & \\
\hline & 14 & 29 & 1,75 & 0,43 & & & \\
\hline \multirow{4}{*}{ PLACERIDESACTIVACIÓN } & 11 & 27 & 2,89 & 0,79 & \multirow{4}{*}{3,113} & \multirow{4}{*}{2,33} & \multirow{4}{*}{0,079} \\
\hline & 12 & 31 & 2,50 & 1.11 & & & \\
\hline & 13 & 30 & 2,34 & 0,76 & & & \\
\hline & 14 & 29 & 2,36 & 0,78 & & & \\
\hline \multirow{4}{*}{ DISPLACER/DESACTIVACIÓN } & 11 & 27 & 1,70 & 0,49 & \multirow{4}{*}{3,113} & \multirow{4}{*}{2,14} & \multirow{4}{*}{0.100} \\
\hline & 12 & 31 & 1,85 & 0,65 & & & \\
\hline & 13 & 30 & 1,68 & 0,67 & & & \\
\hline & 14 & 29 & 1,46 & 0,45 & & & \\
\hline \multirow{4}{*}{ SATISFACCIÓN CON LA VIDA } & 11 & 27 & 21,04 & 5,52 & & & \\
\hline & 12 & 31 & 20,73 & 3,16 & 911 & 000 & ก:จ? \\
\hline & 13 & 30 & 20,52 & 3,76 & $\sqrt{3}, 110$ & 0,30 & $0,0<0$ \\
\hline & 14 & 29 & 21,48 & 3,34 & & & \\
\hline
\end{tabular}

Nota: D.T. = desviación típica; $\mathrm{gl}=$ grados de libertad; sig. = nivel de significación estadistica. 
relaciones entre los dos tipos de afectividad entre sí fue positiva, pero no significativa $(0,16)$, contrariamente a lo esperable desde los modelos que defienden la bipolaridad afectiva. Cuando se toman en cuenta los elementos del afecto más específico, tampoco se obtienen las simetrías entre placer/displacer y activación/desactivación. El placer / activación y el placer/desactivación sí mantienen relaciones positivas y significativas entre sí, como ocurre en mayor medida entre el displacer/activación y displacer/desactivación. Sin embargo, no se encuentran correlaciones negativas y significativas entre supuestos polos afectivos opuestos.

Cuando se analizan las relaciones con el indice de satisfacción con la vida, destaca la independencia entre este índice y la inteligencia, desvinculándose por tanto el bienestar subjetivo del nivel intelectual general. Cuando se toman en cuenta los componentes del afecto, los resultados muestran como la satisfacción vital está positiva y significativamente relacionada con la afectividad positiva y está negativamente relacionada con la afectividad negativa. Tomando en cuenta los elementos desagregados del afecto, lo que parece observarse es que la afectividad positiva se relaciona con la satisfacción cuando se tienen en cuenta en mayor medida los componentes de activación (como alegre y alerta), y la afectividad negativa cuando se tienen en cuenta los elementos de desactivación (como cansado y triste). En todo caso, debe tenerse en cuenta que, si bien es cierto que existen relaciones significativas entre elementos especificos del afecto y la satisfacción con la vida, los coeficientes no son especialmente elevados (el mayor muestra un coeficiente de $-0,37$ ), lo que es indicador de una escasa varianza común compartida.

Estos análisis hacen referencia a la muestra total. Como se ha indicado en la introducción, cabría esperar una cierta pauta evolutiva en este rango de edad, en la medida en que se modifica no solamente esta edad, sino que el rango va de la pubertad a la adolescencia y además existe un cambio en nivel de estudios (de primaria a secundaria) con diferenciaciones en cuanto a ritmo y exigencia escolar y ello, podría promover una modificación en los cuatro grupos de edades. En este sentido, se repitió el análisis anterior para cada uno de los cuatro grupos. En la tabla 3 se resumen los coeficientes obtenidos. 


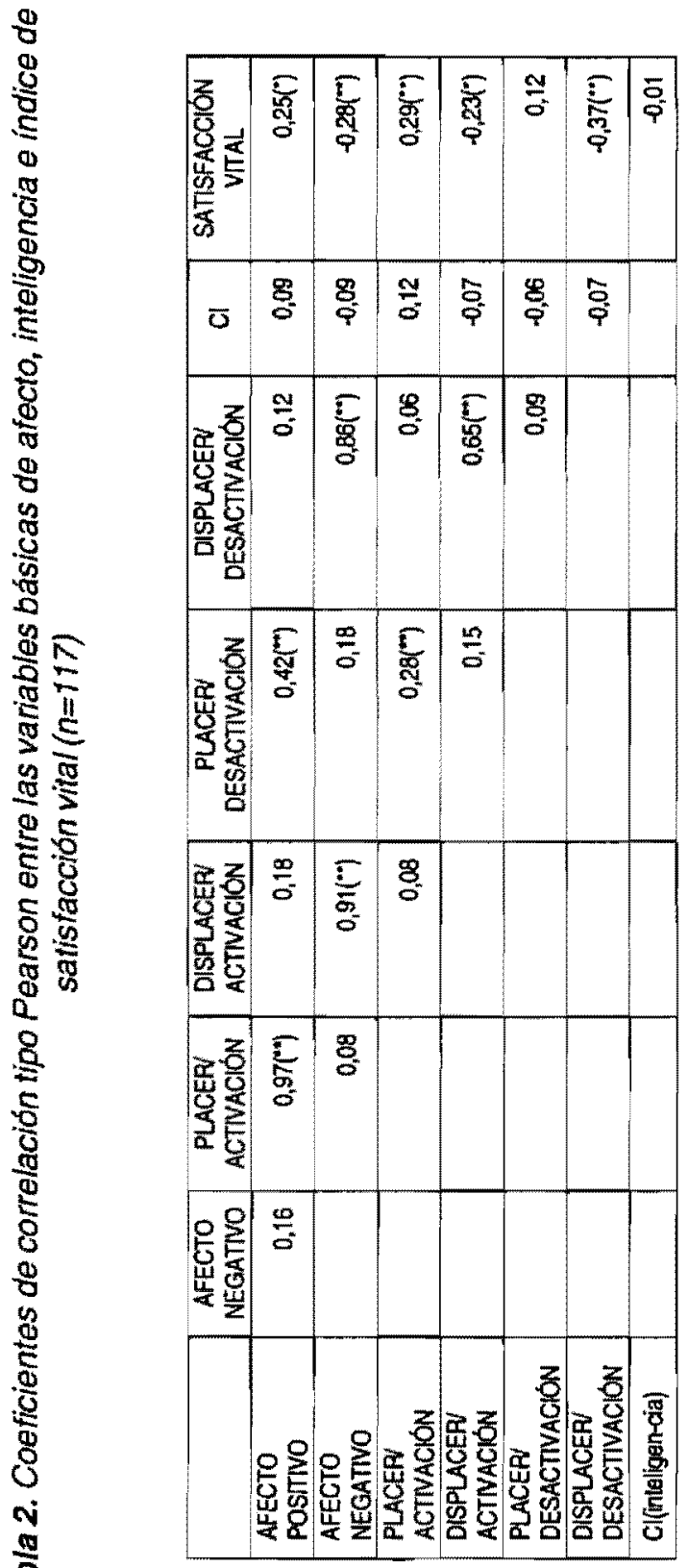

5
0
11
0
$\vdots$
0
0
0
0
0
0
2
2 
Bienestar emocional e inteligencia en la pubertad...

0
0
0
0
0
0
0
$\frac{10}{0}$
0
0

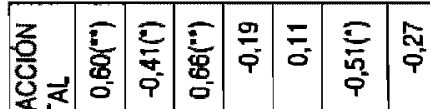

步点

旁

密

$\stackrel{5}{5}$

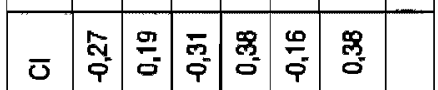

两

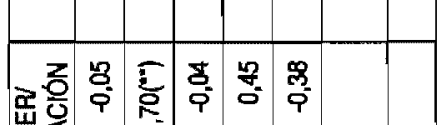

范志

$8 \stackrel{2}{8}$

Y

के

莡 $=$

y

\&

क्षे

空 8

क्ष

논

ธิ

5 온

乌ั

(1)

व

은 뚱

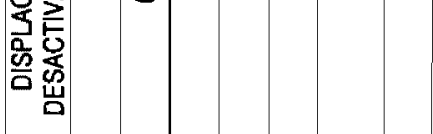

: $\frac{0}{0}$

논

ब

눙

88

$8 \frac{1}{0}$

\&

过

过

这

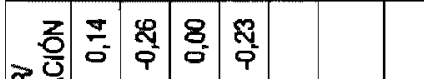

虫案

产

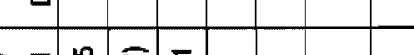

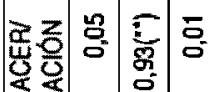

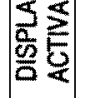

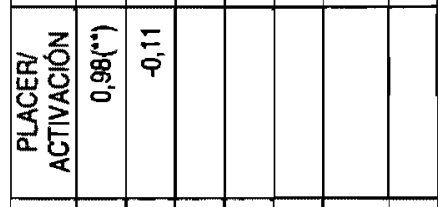

은웡웅

岃焉

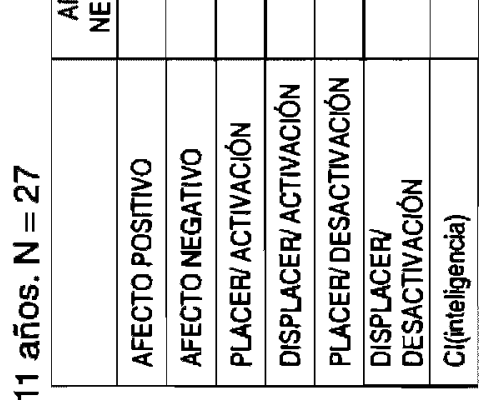

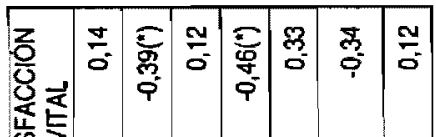

荝

茂

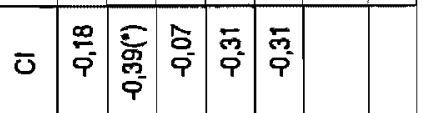

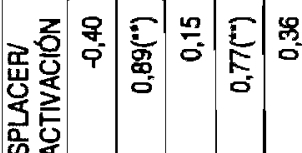

文专

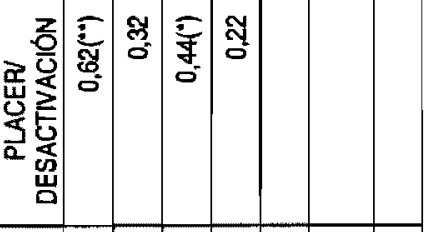

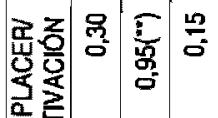

क्ष

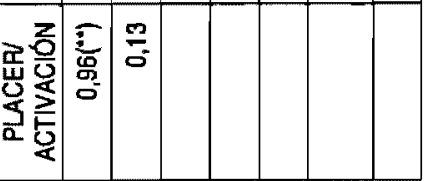

율 $\frac{N}{0}$

出

出昰

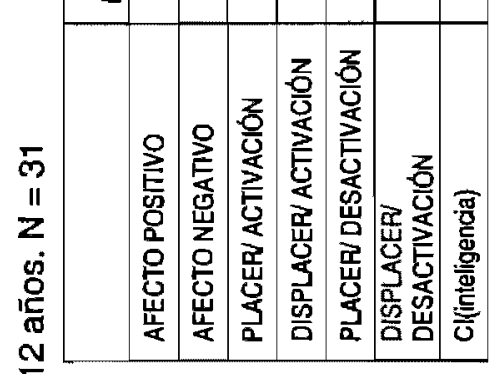



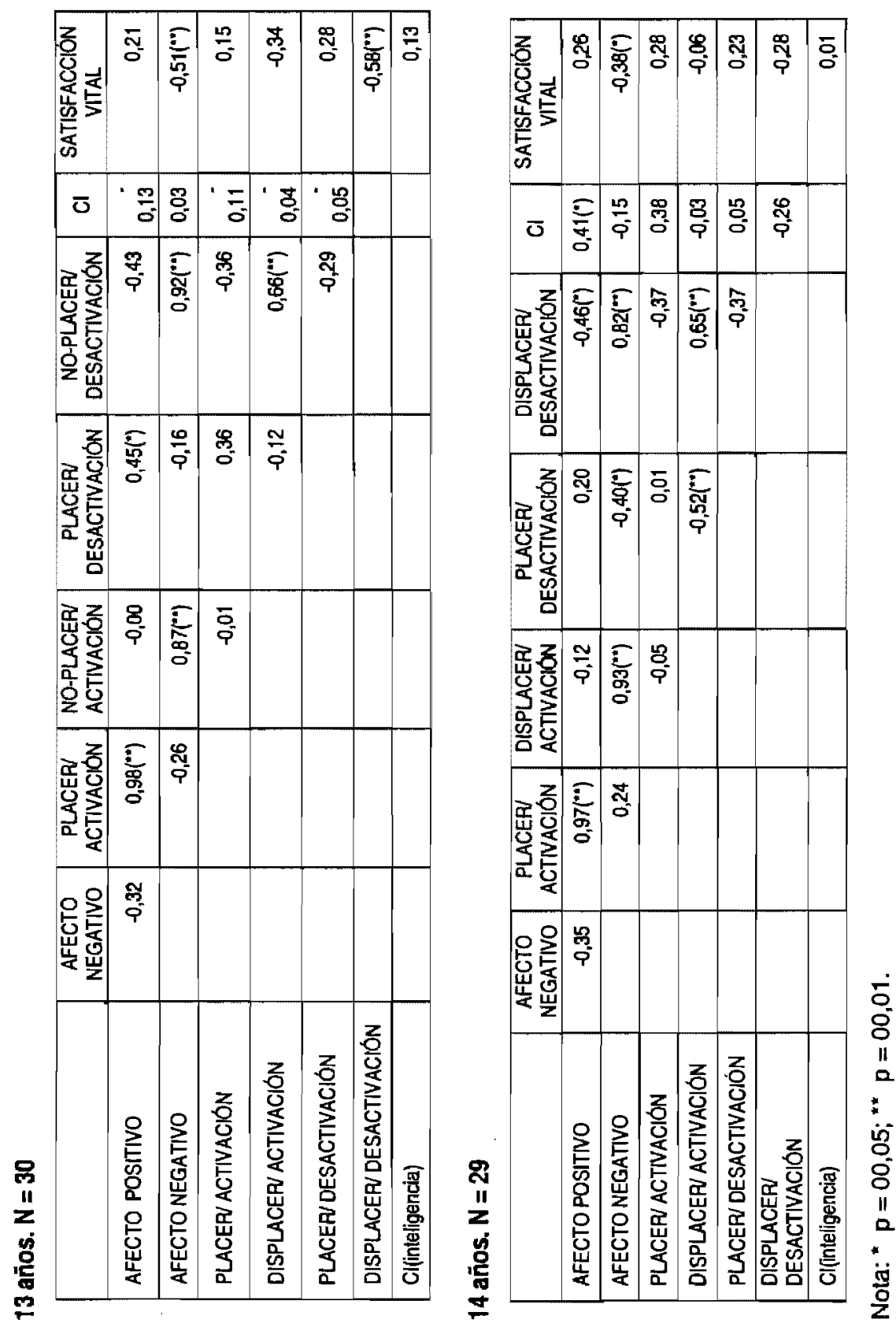
Teniendo en cuenta que el escaso número de participantes por cada grupo de edad hace que estos datos sean esencialmente exploratorios, los resultados muestran una cierta disparidad en función de la edad analizada. Tomando en consideración las relaciones entre inteligencia y los componentes básicos del afecto, se muestra de nuevo una cierta independencia entre ellos, pero también puede observarse cómo la inteligencia parte, en las edades más tempranas, de una relación negativa con la afectividad, disminuyendo progresivamente hasta invertirse la relación a los 14 años, presentando una relación positiva y significativa con la afectividad positiva (de nuevo referida esencialmente a placer/activación).

Las relaciones entre los componentes genéricos del afecto son complejas, pudiéndose observar una cierta independencia entre el afecto positivo y el negativo, relaciones negativas e, incluso, alguna relación positiva. En cualquier caso, son coeficientes no significativos, lo que viene a atestiguar la dificultad de establecer un patrón estable de relaciones entre ambos polos del afecto y, caso de aceptar estos resultados como marcadores, se propondría una ausencia de relación.

Con respecto a las relaciones con la satisfacción vital, la afectividad positiva mantiene relaciones positivas, pero que tienden a disminuir con la edad. Esa relación es negativa con la afectividad negativa y, en este caso, es una relación más estable a lo largo de los cuatro grupos de edades. De nuevo también se observa cómo las relaciones de la satisfacción con el afecto positivo tienen más que ver con los componentes de activación, mientras que las relaciones negativas con el afecto negativo guardan más relación con los componentes de desactivación. Sería el parámetro activación-desactivación el relevante.

En el caso de las relaciones entre la inteligencia y la satisfacción con la vida, los coeficientes obtenidos no son significativos y, en todo caso, se sugiere una evolución, pasando de unas relaciones negativas a unas relaciones de escasa cuantía alrededor de cero $y$, posteriormente, tenderían a presentar relaciones positivas.

Un comentario aparte merecen los resultados sobre afectividad para el subgrupo de 12 años, ya que se comporta de manera distinta a los otros, pudiéndose entrever un papel más importante del polo activación/ desactivación que del polo placer/displacer, si bien el volumen de la muestra no permite generalizaciones claras. 
En todos los casos, como ocurre para los datos globales, las cuantías de las correlaciones suelen ser bajas, dando cuenta de una escasa varianza común explicada, especialmente en el caso de las relaciones con el nivel de satisfacción vital. En este sentido, y tomando como variable a predecir ese nivel de satisfacción, se quiso conocer si tomando todas las variables en conjunto, ese porcentaje de varianza explicada aumentaba, dado que las variables en principio eran aquellas que estarian vinculadas con los modos en el que las personas nos relacionamos con nuestro contexto y entre si, no presentan relaciones altas. El análisis de regresión incluyó, además de la inteligencia, las cuatro medidas desagregadas de la afectividad positiva y negativa, visto el papel diferencial que parecen jugar. En la tabla 4 se resumen los datos y coeficientes obtenidos.

Tabla 4. Análisis de regresión múltiple tomando como variable criterio el nivel de satisfacción vital y como variables a predecir las relacionadas con la afectividad positiva y negativa y el $\mathrm{Cl}(n=117)$

\begin{tabular}{|l|r|r|r|r|}
\hline variables & \multicolumn{1}{c|}{$\beta$} & Error típico & \multicolumn{1}{c|}{$\mathrm{t}$} & significación \\
\hline Constante & 20,33 & 3,20 & 6,36 & 0,00 \\
\hline DISPLACER/DESACTIVACIÓN & $-1,74$ & 0,77 & $-2,26$ & 0,03 \\
\hline PLACER/ACTIVACIÓN & 1,50 & 0,73 & 2,04 & 0,05 \\
\hline DISPLACERIACTIVACION & $-0,21$ & 0,85 & $-0,25$ & 0,81 \\
\hline PLACER/DESACTIVACION & 0,19 & 0,44 & 0,42 & 0,67 \\
\hline CI(inteligencia) & $-0,02$ & 0,02 & $-1,04$ & 0,30 \\
\hline
\end{tabular}

$\hat{a}=$ coeficientes beta; $\mathrm{t}=\mathrm{t}$ de Student.

El análisis de regresión ofreció una función significativa $\left(F_{5,111}=2,63\right.$; $p=0,03$ ). El modelo resultante, sin embargo, sólo ofrece participaciones significativas en la función a las variables de placer/activación y displacer-desactivación (en este caso con una contribución negativa), siendo la varianza total explicada escasa $(R=0,38)$.

Para los cuatro grupos de edades, los resultados generales son homologables sólo en el primer grupo de edad (11 años). Para el resto de las edades las funciones fueron más complejas. Un resumen de los datos generales se recoge en la tabla 5 .

De las cuatro funciones posibles, sólo las dos primeras fueron significativas (para 11 años, $F_{5,21}=8,45, p=0,002$; para 12 años, 
Tabla 5. Resumen de los resultados principales de los análisis de regresión (coeficientes a y probabilidad parcial asociada a cada una de las variables), para cada uno de los grupos de edad, tomando como variable criterio el nivel

de satisfacción vital y como variables a predecir las relacionadas con la afectividad positiva y negativa y la inteligencia fluida

\begin{tabular}{|c|c|c|c|c|c|c|c|c|}
\hline & \multicolumn{2}{|c|}{11 AÑOS } & \multicolumn{2}{|c|}{12 AÑ̃S } & \multicolumn{2}{|c|}{13 AÑOS } & \multicolumn{2}{|c|}{14 AÑOS } \\
\hline & $\beta$ & $p$ & $\beta$ & $p$ & $\beta$ & $p$ & $\beta$ & p \\
\hline Constante & 33,25 & 0,044 & 22,85 & $0, \infty 01$ & 26,84 & 0,001 & 22,85 & 0,001 \\
\hline PLACERIACTIVACIÓN & 5,05 & 0,002 & $-1,35$ & 0,54 & $-0,60$ & 0,68 & $-1,35$ & 0,54 \\
\hline DISPLACERIDESACTIVACIÓN & $-6,67$ & 0,006 & $-0,01$ & 0,99 & $-3,87$ & 0,01 & $-0,01$ & 0,99 \\
\hline DISPLACEPVACTVVACTÓN & 0,54 & 0,756 & 1,84 & 0,38 & 1,97 & 0,31 & 1,84 & 0,38 \\
\hline PLACERDESACTIVACTÓN & $-2,53$ & 0,018 & 0,30 & 0,84 & $-0,09$ & 0,41 & 0,30 & 0,84 \\
\hline a(inteligencia) & $-0,12$ & 0,189 & 0,01 & 0,92 & 0,02 & 0,61 & 0,01 & 0,92 \\
\hline A & \multicolumn{2}{|c|}{0,89} & \multicolumn{2}{|c|}{0,68} & \multicolumn{2}{|c|}{0,67} & \multicolumn{2}{|c|}{0,36} \\
\hline
\end{tabular}

$\mathrm{R}=$ coeficiente de correlación múltiple; $\mathrm{a}=$ coeficientes beta; $\mathrm{p}=$ probabilidad estadistica.

$F_{5,25}=3,09, p=0,03$; para 13 años, $F_{5,24}=2,32, p=0,098$; para 14 años, $F_{5,23}=0,47, p=0,792$ ). La evolución de la varianza común explicada disminuye a medida que se asciende en la edad. Esa disminución es muy llamativa, porque pasa de casi un $80 \%$ a menos del $15 \%$. En la figura 1 representamos esos porcentajes para los cuatro grupos de edades.

Los resultados apuntarían a una progresiva diferenciación y/o a una disminución en la capacidad predictiva de la afectividad más inteligencia (respecto a la satisfacción con la vida) en la adolescencia respecto a la pubertad y, por ello, la idea de que la satisfacción con la vida toma en cuenta más aspectos que la afectividad y la inteligencia, por lo que la idea de bienestar tal y como se operacionaliza para los adultos debería ser revisada con el fin de incorporar otras variables, en la pubertad y la adolescencia.

Los resultados apuntarían a una progresiva diferenciación y/o a una disminución en la capacidad predictiva de la afectividad más inteligencia 
Figura 1. Porcentajes de varianza explicados de la variable satisfacción vital por los análisis de regresión, tomando en cuenta las variables especificas de afecto y la inteligencia

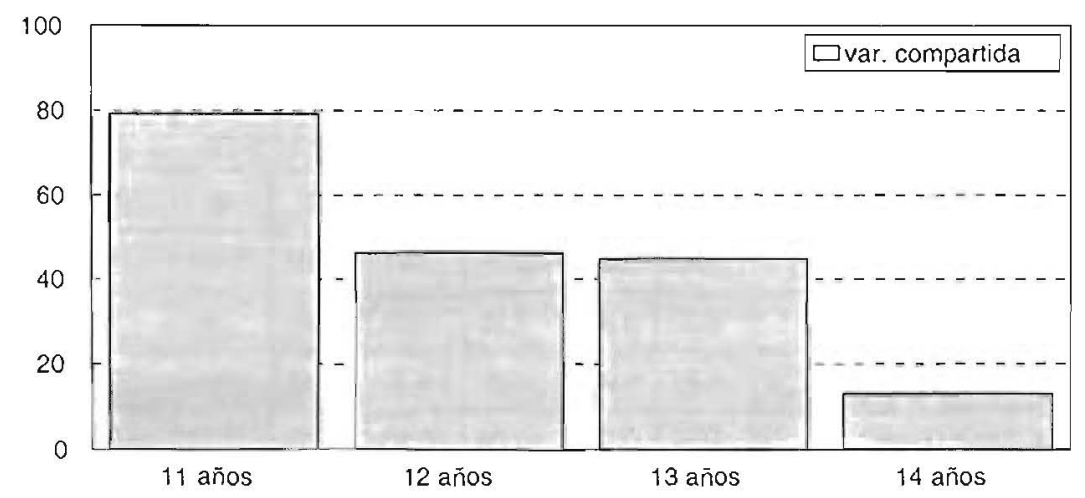

Nota.- Los porcentajes se refieren al cuadrado de la correlación múltiple. Los sombreados cubren la varianza compartida.

(respecto a la satisfacción con la vida) en la adolescencia respecto a la pubertad y, por ello, la idea de que la satisfacción con la vida toma en cuenta más aspectos que la afectividad y la inteligencia, por lo que la idea de bienestar tal y como se operacionaliza para los adultos debería ser revisada con el fin de incorporar otras variables, en la pubertad y la adolescencia.

\section{DISCUSIÓN}

Una primera consideración se refiere a la muestra que ha participado en el estudio: objetivamente se trata de colaboradores voluntarios y el número de participantes en edades distintas permite proponer sugerencias en cuanto a resultados, que deberían ser contrastadas en estudios posteriores con mayor número de muestra en cada grupo de edad.

Un segundo aspecto a considerar, aun dando por buena la estructura de la teoría propuesta acerca del bienestar emocional se refiere a su génesis. Es sensato pensar que los componentes de esta opción deberían estar presentes, y además, guardar las relaciones propuestas, en los estadios evolutivos anteriores. $O$, si no es así, al menos podrían, 
al menos, rastrearse estos elementos en el funcionamiento de los adolescentes. Los resultados que se han presentado ofrecen una imagen un tanto diferente: la satisfacción con la vida parece estar muy relacionada con la afectividad durante la pubertad aunque en la adolescencia deberían ser tomadas en consideración otro tipo de variables (la inteligencia no parece desempeñar función alguna).

Una tercera consideración, más especifica, se refiere a los tres dominios sobre los que versa el trabajo: la afectividad y sus componentes, la relación con la satisfacción con la vida y el papel de la inteligencia.

El más claro de los tres se refiere al papel de la inteligencia. La prueba erripleada se refiere propiamente a la inteligencia que se supone con una cierta base biológica, prácticamente inmodificable y comprometida con eficacia dentro del funcionamiento básicamente académico (aunque escasamente sensible al entrenamiento académico, en opinión de Cattell). Esta inteligencia se presenta, a lo largo de todas las edades, independiente de la afectividad así como de la satisfacción con la vida. Es muy posible que esta independencia se refiera a las "condiciones" de funcionamiento cotidiano y en la que no se exigen grandes esfuerzos ni recursos psicológicos: si la situación de estrés es grande y las tareas a realizar son personalmente comprometidas (como podría suceder en el caso de la toma de decisiones acerca de estudiar o no, presentarse a una prueba o no), es posible que las relaciones fueran otras. El caso es que los resultados muestran con claridad que afectividad y la satisfacción con la vida se presentan como independientes de la inteligencia.

El segundo bloque de variables se refiere a la afectividad a partir de la versión del PANAS para niños y, en su caso, adolescentes. La afectividad positiva y la negativa se presentan como independientes en general. El afecto positivo parece estar más comprometido con el parámetro activación-desactivación, rrientras que en la afectividad negativa tanto displacer como activación parecen desempeñar un papel similar.

El tercer bloque de resultados se refiere a satisfacción con la vida y el bienestar emocional. Cabría esperar teóricamente que en el bienestar emocional no deberían encontrarse presentes elementos de afectividad-emocionalidad displacenteros. De hecho, los resultados alcanzados van en la línea de relaciones positivas entre satisfacción 
con la vida y afectividad y negativas entre satisfacción y afectividad negativa, aunque la cuantía de los coeficientes sugiere que emocionalidad y satisfacción con la vida parecen ser dominios relacionados aunque fácilmente diferenciables (la varianza compartida tiende a ser menos al $10 \%$ ).

Finalmente, los resultados del análisis de regresión por edades (satisfacción con la vida a partir de emocionalidad e inteligencia), aunque tentativos y muy exploratorios, sugeririan una tendencia clara de una progresiva independencia entre estos dominios, independencia que parece suceder entre los 12 y los 13 años, para plasmarse ya en los adolescentes de 14 años. Indirectamente, estos resultados hablarian a favor de una hipótesis genérica de progresiva diferenciaciónindependencia entren domirios psicológicos que se dirigirían a una nueva estructura. Y ello implicaria que la teoria del bienestar emocional a partir de emocionalidad y satisfacción con la vida debería ser tomada con precaución en estas fases evolutivas.

\section{REFERENCIAS BIBLIOGRAFICAS}

Bryant, F. B. \& Veroff, J. (1982).- The structure of psychological wellbeing: A sociohistorical analysis, Journal of Personality and Social Psychology, 43, 653-673.

Cattell, R. B. (1931).- Handbook for the Cattell verbal and non-verbal intelligence tests, London, Barrap Co. (adaptaci 'no española, TEA, Madrid, 1973).

Diener, E. (1984).- Subjective well-being, Psychological Bulletin,95, 542575.

Frida, N. H. (1999).- Emotions and hedonic experience. En D. Kahneman, E. Diener \& N. Schwartz (eds.).- Well-being: The foundations of hedonic psychology,New York, Russell Sage Foundation, 190-210.

Isen, A. M. (2003).- Positive affect as a source of human strength, En L. G. Aspinwall \& U. M. Staudinger (eds).- A Psychology of Human Strengths, Washington D.C., American Psychological Association, 179-196.

Huebner, E. S. (1991).- Initial development of the Student's Life Satisfaction Scale, School Psychology International, 12, 231-240. 
Huebner, E. S. (1994).- Preliminary development and validation of a multidimensional life satisfaction scale for children, Psychological Assessment, 6, 149-158.

Jahoda, M. (1958).- Current concepts of positive mental health, New York, Basic Books.

Keyes, C.L.M. (1998).- Social well-being, Social Psychology Quarterly, 61, 121-140.

Keyes, C.L.M. \& Magyar-Moe, J.L.(2003).- The measurement and utility of adult subjective well-being, En S. J. Lopez \& C. R. Snyder (eds).Positive psychological assessment. $A$ handbook of models and measures, Washington, American Psychological Association, 411- 425. Keyes, C. L. M. \& Riff, C. D. (2000).- Subjective change and mental health: A self concept theory, Social Psychology Quarterly, 63, 264279.

Larsen, R. J. \& Diener, E. (1992).- Promises and problems with the circumplex of emotion. En M. S. Clark (ed).- Review of personality and social psychology: Emtion, vol. 13, 25-59, Newbury Park, CA, Sage.

Laurent, J., Catanzaro, S., Joiner, Jr., T., Rudoph, K., Potter, K., Lambert, S., Osborne, L. \& Gathright, T. (1999).- A measure of positive and negative affect for children: scale development and preliminary validation, Psychological Assessment, 11, 326-338.

Lucas, R. E., Diener, E. \& Larsen, R. J. (2003).- Measuring positive emotions. En S. J. Lopez \& C. R. Snyder (eds.).- Positive psychological assessment. A handbook of models and measures, Washington, American Psychological Association, pp. 201- 218.

Lucas, R.E., Diener, E. \& Suh, E. (1996).- Discriminant validity of wellbeing measures, Journal of Personality and Social Psychology, 71, 616-628.

Ortony, A. \& Turner, T. J. (1990).- What's basic about basic emotions? Psychological Review, 97, 315-331.

Pelechano, V. (1996).- Una introducción al modelo de parámetros en psicología. En V. Pelechano (dir).- Psicología de la personalidad.I.Teorias, Barcelona, Ariel, 337-368.

Pelechano,V. (1999).- Habilidades interpersonales: antecedentes, sentido y operacionalización, Análisis y modificación de Conducta, 25, 171-195. 
Pelechano, V. (2000).- Psicología sistemática de la personalidad, Ariel, Barcelona.

Pestana, J., Rosich, M. \& Codina, N. (2003).- Life, environment, self and sport satisfaction, Medio ambiente y comportamiento humano, 4,71-79.

Russell, J. A. (1980).- A circumplex model of affect, Journal of Personality and Social Psychology,39, 1161-1178.

Russell, J. A. \& Feldman Barrett, L. (1999).- Core affect, prototypical emotional episodes, and other things called emotion: Dissecting the elephant, Journal of Personality and Scoail Psychology, 76, 805-819. Ryff, C.D. \& Keyes, C.L.M.(1995).- The structure of psychological wellbeing revisited, Journal of Personality and Social Psychology, 69, 1069-1081.

Sandin, B. (2003).- Escalas PANAS de afecto positive y negativo para niños y adolescents (PANASN), Revista de Psicopatología y Psicología Clínica, 8, 173-182.

Schimmack, U. \& Grob, A. (2000).- Dimensional models of core affect: A quantitative comparison by means of structural equation modelling, European Journal of Personality, 14, 325-345.

Shmotkin, D. (1998).- Declarative and differential aspects of subjective well-being and implications for mental health in later life. En J. Lomranz (ed.).- Hanbook of aging and mental health: An integrative approach, New York, Plenum Press, 15-43.

Sternberg, R. J. (2003).- Driven to despair: Why we need to redefine the concept and measurement of intelligence, En L. S. Aspinwall \& U.M. Staudinger (eds).- A Pschology of Human Strenghts, Washington D.C., American Psychological Association, 319-330.

Watson, D., Clark, L. \& Tellegen, A. (1988).- Development and validation of brief measures of positive and negative affect: the PANAS scales, Journal of Personality and Social Psychology, 54, 1063-1070.

Watson, D. \& Tellegen, A. (1985).- Toward a consensual structure of mod, Psychological Bulletin, 98, 219-235.

Watson, D., Wiese, D., Vaidya, J. \& Tellegen, A. (1999).- The two general activation systems of affect. Structural findings, evolutionary considerations, and psychobiological evidence, Journal of Personality and Social Psychology, 76, 820-838.

Zautra, A. J., Potter, P. T. \& Reich, J. W. (1997).- The independence of 
affects is context-dependent: An integrative model of the relationship of positive and negative affect. En K. W. Schaie \& M. P. Lawton (eds).- Annual Review of gerontology and geriatrics, vol. 17, 75103, New York, Springer. 